\title{
Pretilt Angle of Liquid Crystals and Liquid-Crystal Alignment on Microgrooved Polyimide Surfaces Fabricated by Soft Embossing Method
}

\author{
Da-Ren Chiou and Li-Jen Chen* \\ Department of Chemical Engineering, National Taiwan University, Taipei 10617, Taiwan \\ Chein-Dhau Lee \\ Materials and Chemical Research Laboratories, Industrial Technology Research Institute, Hsinchu 31015, \\ Taiwan
}

Received June 29, 2006. In Final Form: August 23, 2006

\begin{abstract}
In this study, the soft embossing method is proposed to fabricate periodical microgrooved structure on polyimide surfaces. These microgrooved polyimide surfaces are assembled to form liquid-crystal cells. It is found that the director of liquid crystals uniformly aligns along the groove direction even when the groove width is as high as $3 \mu \mathrm{m}$. The anchoring energy of these microgrooved polyimide surfaces is higher than that of the typical rubbed surfaces. The pretilt angle of liquid crystals is adjusted by tuning the surface polarity of the polyimide alignment layer, which is identified by the advancing contact angle of water. The surface polarity of polyimide alignment layers is manipulated by simply mixing two kinds of polyimide: a more hydrophilic one and a more hydrophobic one. It is found that the pretilt angle of liquid crystals increases along with the advancing contact angle of water on the alignment layer under the condition of a fixed surface topography.
\end{abstract}

\section{Introduction}

In the liquid-crystal display industry, the unidirectional mechanical rubbing process on polymer-coated substrates with a velvet cloth is almost exclusively applied to align liquid crystals. The rubbing process induces microgrooves on the polymer surfaces, and the liquid-crystal molecules would align along the direction of the microgrooves. On the other hand, the rubbing process also creates debris and electrostatic charge that deteriorate the display quality. ${ }^{1}$ To resolve these problems, nonrubbing methods have been intensively explored, such as ultraviolet ${ }^{2}$ or ion beam $^{3}$ irradiation. These methods are still subject to the reliance on polymer substances. In this study, a nonrubbing soft embossing method is proposed to fabricate reliable periodical microgrooves on the polymer-coated substrates to induce the liquid-crystal alignment without the disadvantages of the rubbing process. This soft embossing method has been successfully applied to fabricate silica grating substrates for liquid-crystal alignment by using sol-gel precursor recently. ${ }^{4}$

In addition to the uniform alignment of liquid-crystal molecules, an appropriate pretilt angle of liquid-crystal molecules is necessary for the twisted nematic liquid-crystal displays to prevent from

* Author to whom correspondence should be addressed. E-mail: ljchen@ ntu.edu.tw.

(1) van Haaren, J. Nature (London) 2001, 411, 29.

(2) (a) Gibbons, W. M.; Shannon, P. J.; Sun, S. T.; Swetlin, B. J. Nature 1991, 351, 49. (b) Shannon, P. J.; Gibbons, W. M. Nature 1994, 368, 532. (c) Schadt M.; Seiberle, H.; Schuster, A. Nature 1996, 381, 212. (d) Behdani, M.; Keshmiri, S. H.; Soria, S.; Bader, M. A.; Ihlemann, J.; Marowsky, G.; Rasing, Th. Appl. Phys. Lett. 2003, 82, 2553. (e) Ichimura, K.; Suzuki, Y.; Seki, T.; Hosoki, A.; Aoki, K. Langmuir 1988, 4, 1214. (f) Fang, J. Y.; Chen, M.-S.; Shashidhar, R. Langmuir 2001, 17, 1549.

(3) (a) Stöhr, J.; Samant, M. G.; Lüning, J.; Callegari, A. C.; Chaudhari, P.; Doyle, J. P.; Lacey, J. A.; Lien, S. A.; Purushothaman, S.; Speidell, J. L. Science 2001, 292, 2299. (b) Chaudhari, P.; Lacey, J.; Doyle, J.; Galligan, E.; Lien, S. A.; Callegari, A.; Hougham, G.; Lang, N. D.; Andry, P. S.; John, R.; Yang, K.-H.; Lu, M.; Cai, C.; Speidell, J.; Purushothaman, S.; Ritsko, J.; Samant, M.; Stohr, J.; Nakagawa, Y.; Katoh, Y.; Saitoh, Y.; Sakai, K.; Satoh, H.; Odahara, S.; Nakano, H.; Nakagaki, J.; Shiota, Y. Nature 2001, 411, 56.

(4) Chiou, D.-R.; Yeh, K.-Y.; Chen, L.-J. Appl. Phys. Lett. 2006, 88, 133123. reverse tilt disclinations upon exerting an external electric field. Recently, the control of pretilt angle of liquid-crystal molecules has been intensively investigated. ${ }^{5-16}$ It was pointed out that the rubbing process induces polar functional groups and repeating units to reorient out of the plane of the alignment film and nonpolar aliphatic side chains to partially reorient toward the bulk of the alignment film..$^{5-7,15}$ These studies demonstrate that the pretilt angle of liquid-crystal molecules increases along with the rubbing strength. ${ }^{5-9}$ The pretilt angle can also be increased by simply introducing long, linear alkyl side chains or other nonpolar groups to the polyimide alignment layers. ${ }^{5,10-12}$ On the other hand, applying the surface treatment of UV exposure or $\mathrm{O}_{2}$ plasma to the polyimide alignment layers would increase the surface polarity and decrease the pretilt angle of liquid-crystal molecules. , $^{2}, 16$ All these previous studies implied that the pretilt angle is strongly related to the surface polarity. In this study, the advancing contact angle of water is used as an index of the surface polarity. The soft embossing method is applied to fabricate the microgrooved polymer surfaces to align the liquid-crystal molecules. The surface polarity of polyimide alignment layers is manipulated by simply mixing two kinds of polyimide: one is more hydrophilic and the

(5) Lee, K.-W.; Lien, A.; Stathis, J. H.; Paek, S.-H. Jpn. J. Appl. Phys. 1997 36, 3591 .

(6) Paek, S.-H.; Durning, C. J.; Lee, K.-W.; Lien, A. J. Appl. Phys. 1998, 83, 1270.

(7) Sinha, G. P.; Wen, B.; Rosenblatt, C. Appl. Phys. Lett. 2001, 79, 2543.

(8) Becker, M. E.; Kilian, R. A.; Kosmowski, B. B.; Mlynski, D. A. Mol. Cryst. Liq. Cryst. 1986, 132, 167. 23.

(10) Ha, K.; West, J. L. Liq. Cryst. 2004, 31, 753.

(11) Seo, D.-S.; Kobayashi, S.; Nishikawa, M.; Yabe, Y. Jpn. J. Appl. Phys. 1996, 35, 3531 .

(12) Seo, D.-S.; Kobayashi, S. Liq. Cryst. 2000, 27, 883

(13) Newsome, C. J.; O'Neill, M. J. Appl. Phys. 2002, 92, 1752.

(14) Hatoh, H.; Shohara, K.; Kinoshita, Y.; Ookoshi, N. Appl. Phys. Lett. 1993, 63, 3577.

(15) Lee, K.-W.; Paek, S.-H.; Lien, A.; Durning, C. J.; Fukuro, H. Macromolecules, 1996, 29, 8894.

(16) Moore, J. A.; Dasheff, A. N. Chem. Mater. 1989, 1, 163. 

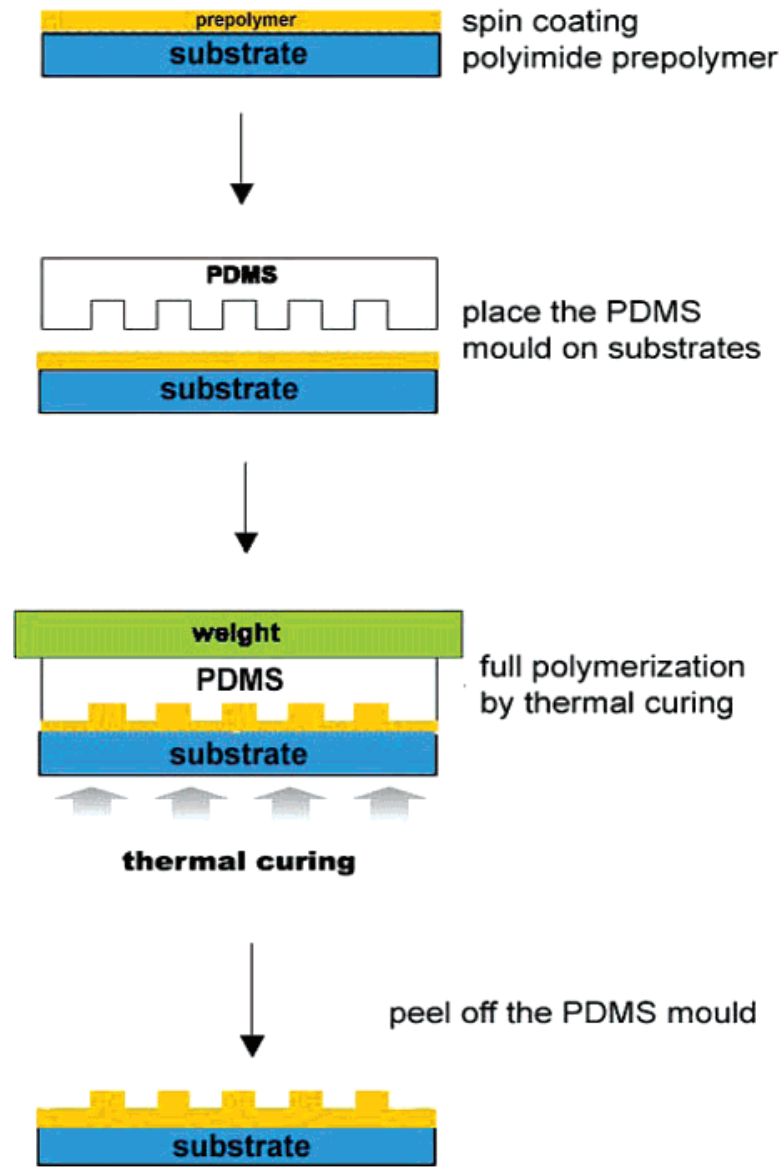

Figure 1. 1. The schematic illustration of the soft embossing method.

other one is more hydrophobic. Our experimental results show that the pretilt angle is indeed adjustable by the modification of the surface polarity of the alignment layer. In addition, the pretilt angle increases along with an increase in the surface polarity of the alignment layer.

\section{Experimental Section}

Materials. Octadecyltrichlorosilane (OTS), $\mathrm{H}_{2} \mathrm{SO}_{4}$ (98\%), and the nematic liquid-crystal 4- $n$-pentyl-4'-cyanobiphenyl (5CB) were obtained from Aldrich. $\mathrm{H}_{2} \mathrm{O}_{2}(30 \%)$ and dichloromethane $(99 \%)$ were purchased from Merck. Poly(dimethylsiloxane) (PDMS) SylgardTM184 was obtained from Dow Corning Co. Polyimide prepolymers of planar alignment PIA-5310 and of vertical alignment JSR-2021 were obtained from, respectively, Chisso and Japan Synthetic Rubber Corporation. All these chemicals were used as received. The microscope glass slides (FEA) were cleaned by piranha solution [a mixture 7:3 (v/v) of $98 \% \mathrm{H}_{2} \mathrm{SO}_{4}$ and $30 \% \mathrm{H}_{2} \mathrm{O}_{2}$ ] at 120 ${ }^{\circ} \mathrm{C}$ for $30 \mathrm{~min}$ before use.

Fabrication of Microgrooved Polymeric Surfaces. We first fabricated the patterned silicon masters either by photolithography or by the electron-beam method. Then, the masters were dipped into OTS solutions to minimize the adhesion between PDMS mold and the patterned silicon masters. After the pretreatment of these patterned silicon masters, the mixture of the PDMS prepolymer and the curing agent (10:1 by weight) was poured onto the patterned silicon masters. After thermal curing at $60{ }^{\circ} \mathrm{C}$ for $12 \mathrm{~h}$, the patterned PDMS molds were obtained by peeling off the molds from the silicon masters. Next, the soft embossing technique was applied to fabricate the microgrooved polymeric surfaces. Figure 1 schematically illustrates the soft embossing process. A layer of liquid polyimide prepolymer was spin-coated onto the clean glass substrate. Then, the PDMS mold with a microgrooved structure was embossed on this substrate and was followed by the thermal curing process: prebaking at 90 ${ }^{\circ} \mathrm{C}$ for $10 \mathrm{~min}$ and then postbaking at $220{ }^{\circ} \mathrm{C}$ for $30 \mathrm{~min}$. After (a)

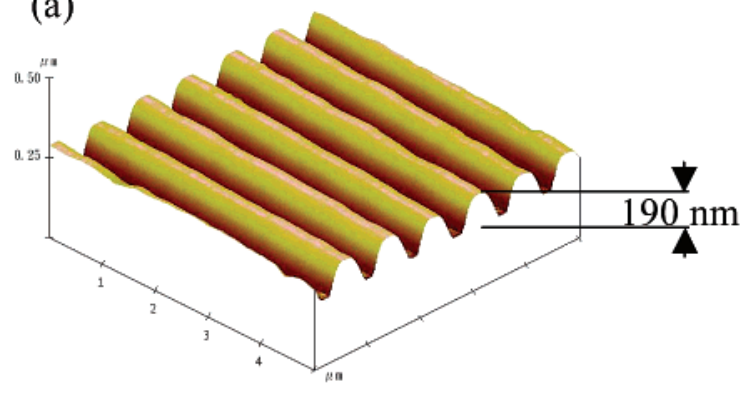

(b)

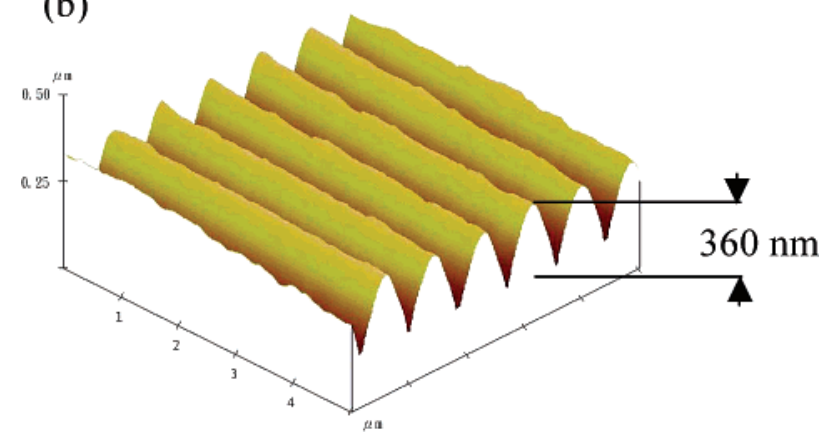

Figure 2. 2. The atomic force microscopic (AFM) images of the microgrooved polyimide surface with $840-\mathrm{nm}$ groove period at two different groove depths: (a) $190 \mathrm{~nm}$ and (b) $360 \mathrm{~nm}$.

peeling off the PDMS mold, the microgrooved structure was fabricated on the polyimide surface. The surface topology was no different whether the PDMS mold was peeled off before or after the postbaking process.

Measurement of the Advancing Contact Angle of Water. The advancing contact angle measurement was performed by a homemade enhanced video-microscopy system incorporated with a digital image analysis. The details of the methodology and its experimental setup can be found elsewhere. ${ }^{17}$ The accuracy of the advancing contact angle measurements is better than $\pm 0.1^{\circ}$, even for small angles. All the advancing contact angle measurements in this work were performed on the flat polyimide surfaces without microgrooves.

Images by Using Atomic Force Microscope (AFM). The contact mode AFM (Nanoscope IIIa, Digital Instrument, Santa Barbara) was used to explore the surface topography of the microgrooved polymer surfaces. Silicon nitride tips (Digital Instrument) with a spring constant of $0.06 \mathrm{~N} / \mathrm{m}$ were used to image samples under ambient conditions.

Assembly of Liquid-Crystal Cells. A liquid-crystal cell was assembled by two microgrooved polymeric surfaces with parallel groove direction. The two substrates were kept apart by inserting $10-\mu \mathrm{m}$ Mylar films (DuPont Films) along the two longer edges. The filling of $5 \mathrm{CBs}$ was by capillarity. After the $5 \mathrm{CB}$ was filled, the edges of the cells were sealed by glues. Next, the cells were heated to about $40{ }^{\circ} \mathrm{C}$ for $5 \mathrm{~min}$ to reach the isotropic phase of $5 \mathrm{CBs}$ and then were cooled at room temperature to get into the nematic state.

Analysis of Optical Textures. A crossed polarized optical microscope (Zeiss) was used to observe the texture of the liquidcrystal cells. A digital camera (Canon) attached to the microscope was used to capture the images of the optical appearance of the liquid-crystal cells.

Measurement of Pretilt Angle of Liquid-Crystal Molecules. In this study, the pretilt angle was measured by the crystal-rotation method $^{18}$ (Autronic-Melchers, TBA 105). All the substrates with a microgrooved pattern of 360-nm groove width, 480-nm line width, and 360-nm groove depth were used to assemble the liquid-crystal cell with a cell gap of $25 \mu \mathrm{m}$ for the pretilt angle measurement.

(17) Yeh, M.-C.; Chen, L.-J.; Lin, S.-Y.; Hsu, C.-T. J. Chin. Inst. Chem. Eng. 2001, 32, 109

(18) Baur, G.; Wittwer, V.; Berreman, D. W. Phys. Lett. 1976, 56A, 142. 
(a)

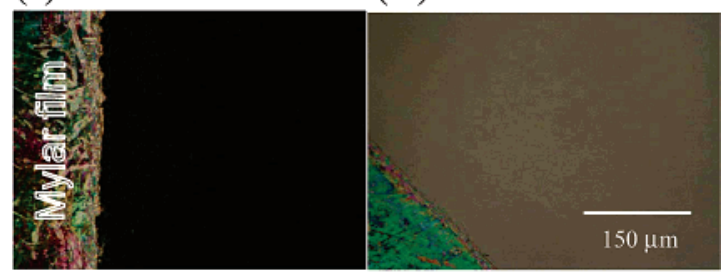

(b)

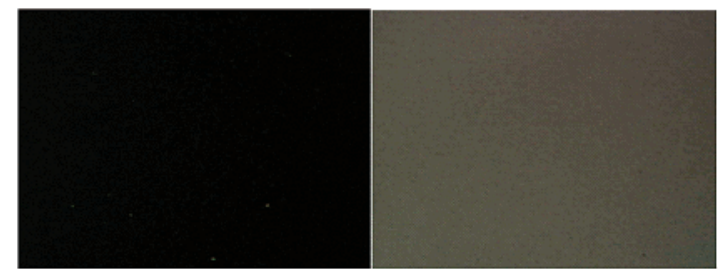

(c)

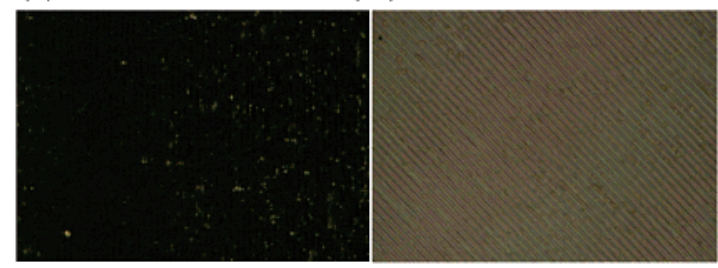

(d)

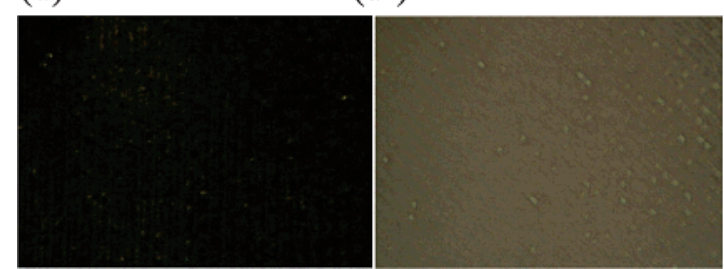

Figure 3. 3. Optical micrographs of liquid-crystal alignment on the microgrooved polyimide surfaces taken between crossed polarizers. (a and $\mathrm{a}^{\prime}$ ) line width $a=0.48 \mu \mathrm{m}$, groove width $b=0.36 \mu \mathrm{m}$, and groove depth $c=0.36 \mu \mathrm{m}$; (b and $\mathrm{b}^{\prime}$ ) $a=1.42 \mu \mathrm{m}, b=2.42 \mu \mathrm{m}$, and $c=0.74 \mu \mathrm{m}$; (c and $\left.\mathrm{c}^{\prime}\right) a=4.44 \mu \mathrm{m}, b=5.27 \mu \mathrm{m}$, and $c=$ $0.32 \mu \mathrm{m} ;\left(\mathrm{d}\right.$ and $\left.\mathrm{d}^{\prime}\right) a=9.28 \mathrm{~mm}, b=10.2 \mu \mathrm{m}$, and $c=0.36 \mu \mathrm{m}$. All the micrographs on the left-hand side, a, b, c, and d, are taken with the groove direction parallel to one of the polarizer axes, and all the micrographs on the right-hand side, $\mathrm{a}^{\prime}, \mathrm{b}^{\prime}, \mathrm{c}^{\prime}$, and $\mathrm{d}^{\prime}$, are taken with the groove direction at $45^{\circ}$ to each polarizer axis.

\section{Results and Discussion}

The AFM images of the polyimide alignment layer with periodical microgrooves fabricated by the soft embossing method are shown in Figure 2. All the edges are not sharp but are rounded in every surface relief because of the slight shrinkage after solvent evaporation. This patterning technique demonstrates good pattern fidelity for the fabrication of large-area microstructures. There is no need of extreme high pressures as used by nanoimprint lithography. ${ }^{19}$ The alignment of the $5 \mathrm{CB}$ molecules in the liquidcrystal cell is observed by optical polarized microscopy with crossed polarizers. Figure 3 shows the optical micrographs taken from the liquid-crystal cells assembled by using the substrates of four different microgrooved structures. The liquid-crystal molecules align planarly on the microgrooved polyimide PIA5310 surfaces along the groove direction and even the groove width is up to $2.4 \mu \mathrm{m}$, as shown in Figure $3 \mathrm{a}$ and $3 \mathrm{~b}$. When the groove width is increased to $5 \mu \mathrm{m}$ or $10 \mu \mathrm{m}$, the liquid-crystal alignment along the groove direction is not uniform anymore

(19) Chou, S. Y.; Krauss, P. R.; Renstrom, P. J. Science 1996, 272, 85.

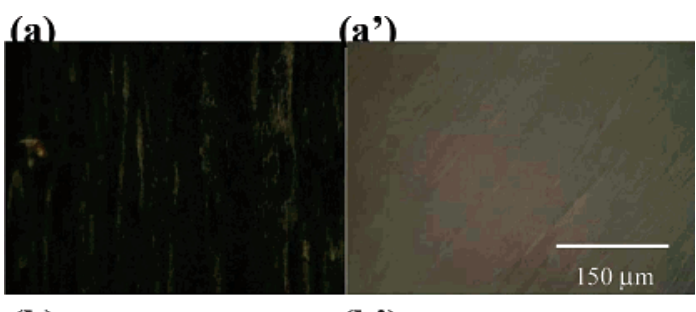

(b)

(b')

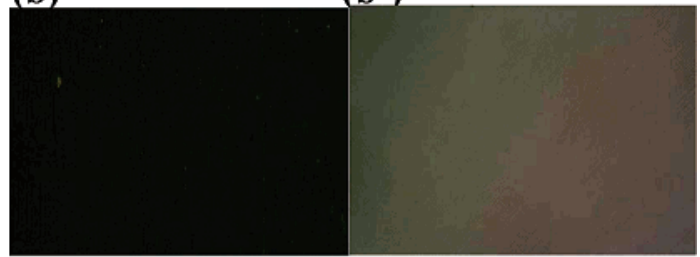

(c)

(c')

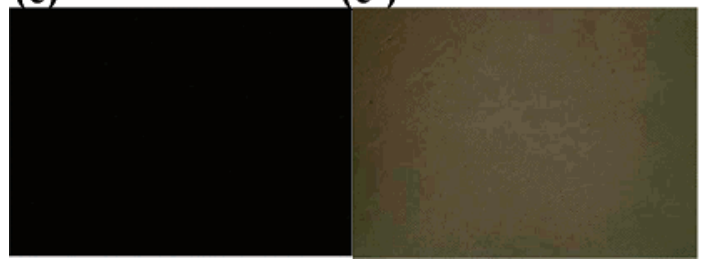

Figure 4. 4. Optical micrographs of liquid-crystal alignment taken between crossed polarizers for the patterned polyimide surfaces with $6-\mu \mathrm{m}$ groove period and $3-\mu \mathrm{m}$ groove width at three different groove depths: $60 \mathrm{~nm}$ (a and $\left.\mathrm{a}^{\prime}\right), 330 \mathrm{~nm}$ (b and b'), and $1630 \mathrm{~nm}$ (c and $\mathrm{c}^{\prime}$ ). All the micrographs on the left-hand side, a, b, and c, are taken with the groove direction parallel to one of the polarizer axes, and all the micrographs on the right-hand side, $a^{\prime}, b^{\prime}$, and $c^{\prime}$, are taken with the groove direction at $45^{\circ}$ to each polarizer axis.

Table 1. Geometric Effect of the Polyimide PIA-5310 Microgrooved Surfaces on the Anchoring Energy

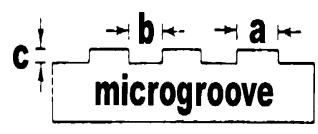

\begin{tabular}{cccc}
\hline $\begin{array}{c}\text { line width, } \\
a(\mathrm{~nm})\end{array}$ & $\begin{array}{c}\text { groove width, } \\
b(\mathrm{~nm})\end{array}$ & $\begin{array}{c}\text { groove depth, } \\
c(\mathrm{~nm})\end{array}$ & $\begin{array}{c}\text { anchoring energy } \\
\left(\mathrm{J} / \mathrm{m}^{2}\right)\end{array}$ \\
\hline $480 \pm 10$ & $360 \pm 10$ & $190 \pm 10$ & $2.43( \pm 0.26) \times 10^{-5}$ \\
$480 \pm 10$ & $360 \pm 10$ & $360 \pm 30$ & $11.1( \pm 5.6) \times 10^{-5}$ \\
$800 \pm 20$ & $610 \pm 20$ & $190 \pm 10$ & $1.74( \pm 0.49) \times 10^{-5}$ \\
$800 \pm 10$ & $610 \pm 20$ & $350 \pm 40$ & $7.11( \pm 4.06) \times 10^{-5}$ \\
$910 \pm 100$ & $1000 \pm 40$ & $660 \pm 20$ & $2.20( \pm 0.40) \times 10^{-5}$ \\
$910 \pm 20$ & $1000 \pm 30$ & $1030 \pm 20$ & $3.91( \pm 0.62) \times 10^{-5}$ \\
$1420 \pm 100$ & $2420 \pm 80$ & $740 \pm 50$ & $2.40( \pm 0.00) \times 10^{-5}$ \\
$1420 \pm 40$ & $2420 \pm 30$ & $1230 \pm 130$ & $4.42( \pm 0.79) \times 10^{-5}$
\end{tabular}

and there are microdomains, as shown in Figure $3 \mathrm{c}$ and $3 \mathrm{~d}$. It is found that the smaller is the spatial period of the microgroove, the more effective is the liquid-crystal alignment along the groove direction.

According to the minimization of the elastic energies for nematic liquid crystals, Berreman ${ }^{20}$ proposed that the groove effect is the primary factor to induce the alignment of liquidcrystal molecules along the rubbing direction. It had also been shown that the rubbing process realigns the polyimide main chains along the rubbing direction that induces the liquid-crystal alignment rather than the rubbing groove effect. ${ }^{21}$ Furthermore, the modern popular nonrubbing methods, such as the photoinduced liquid-crystal alignment by polarized light exposure, ${ }^{22}$ also confirm that the realignment of the polymer main chains

(20) (a) Berreman, D. W. Phys. Rev. Lett. 1972, 28, 1683. (b) Berreman, D. W. Mol. Cryst. Liq. Cryst. 1973, 23, 215.

(21) Castellano, J. A. Mol. Cryst. Liq. Cryst. 1983, 94, 33.

(22) O’Neill, M.; Kelly, S. M. J. Phys. D: Appl. Phys. 2000, 33, R67. 
Table 2. The Advancing Contact Angles $\left(\theta_{\mathrm{a}}\right)$ of Water and the Corresponding Pretilt Angles $\left(\boldsymbol{\theta}_{\mathrm{p}}\right)$ of 5 CBs on the Pure Polyimide (PI) Surfaces and on the Surfaces Prepared from the Mixtures of the Prepolymers of PIA-5310 and JSR-2021 at Different Volume Ratios of PIA-5310/JSR-2021

\begin{tabular}{|c|c|c|c|c|c|c|c|c|c|c|c|c|}
\hline \multirow[b]{2}{*}{ PI } & \multirow[b]{2}{*}{ JSR-2021 } & \multicolumn{10}{|c|}{ volume ratio $(\mathrm{PIA}-5310 / \mathrm{JSR}-2$ 021) $=$} & \multirow[b]{2}{*}{ PIA-5310 } \\
\hline & & $<100$ & 100 & 175 & 250 & 375 & 500 & 625 & 750 & 875 & 1000 & \\
\hline$\theta_{\mathrm{a}}$ & $99^{\circ}$ & & $95^{\circ}$ & $93^{\circ}$ & $89^{\circ}$ & $82^{\circ}$ & $80^{\circ}$ & $78^{\circ}$ & $74^{\circ}$ & $73^{\circ}$ & $70^{\circ}$ & $67^{\circ}$ \\
\hline$\theta_{\mathrm{p}}$ & $90.0^{\circ}$ & $90.0^{\circ}$ & $89.9^{\circ}$ & a & $a$ & $14.9^{\circ}$ & $11.5^{\circ}$ & $8.4^{\circ}$ & $2.3^{\circ}$ & $0.8^{\circ}$ & $0.7^{\circ}$ & $0.3^{\circ}$ \\
\hline
\end{tabular}

a Poor alignment.

dominates the liquid-crystal alignment. It remains a controversial problem of whether the surface topography or the polymer backbones dominates the alignment of nematic liquid crystals. It is obvious that the soft embossing method does not reorient the polyimide main chains, as the rubbing process does. As a consequence, the groove effect should be the sole factor that induces the alignment of the liquid-crystal molecules in this work. Especially, there is a strong long-range ordering within the liquid-crystal molecules when they are confined in the grooves and even the width is up to $2.4 \mu \mathrm{m}$, as shown in Figure 3.

The anchoring ability of the microgrooved polyimide PIA5310 surface is further examined by measuring the anchoring energy of liquid crystals on the surface. The anchoring energy is an index of the interaction energy between the alignment layer and the liquid-crystal molecules. Rubbed polyimide surface is known to strongly anchor the liquid-crystal molecules in the rubbing direction and is used as a reference surface. The liquidcrystal cell with a cell gap of $10 \mu \mathrm{m}$ is assembled by two alignment surfaces perpendicular to each other: one patterned polyimide surface with microgrooves and one conventionally rubbed polyimide counter plate. The cell rotation method ${ }^{23}$ is applied to determine the surface anchoring energy by determining the twist angle $\phi$ of the liquid-crystal molecules in the liquid-crystal cell according to the following equation.

$$
W_{\phi}=\frac{2 K_{22} \phi}{d \sin 2 \phi}
$$

where $d$ is the cell gap $(10 \mu \mathrm{m})$ and $K_{22}$ is the twist elastic constant of the liquid crystal $\left(3.9 \times 10^{-12} \mathrm{~N}\right.$ for 5 CBs used in this work).

Table 1 lists the anchoring energies for the polyimide PIA5310 alignment layers at different microgrooved structures. It is found that the microgrooved polyimide surfaces have higher anchoring energies than that of the conventional rubbed polyimide surfaces, $1.68( \pm 0.33) \times 10^{-5} \mathrm{~J} / \mathrm{m}^{2}$ in this study. It is believed that the reason for higher anchoring energy is simply due to the large groove depth of the microgrooved polyimide surfaces compared to that of the rubbed ones. For the rubbed polyimide surfaces used in this work, the groove width and depth are 30 and $4 \mathrm{~nm}$, respectively. Consider a condition of a fixed groove depth, say $190 \mathrm{~nm}$. The anchoring energy for the system of $360-\mathrm{nm}$ groove width is larger than that of $610-\mathrm{nm}$ width, as shown in Table 1. That is, the anchoring energy of the microgrooved polyimide surfaces increases along with a decrease in the groove width for those grooves with a same groove depth. Consequently, the liquid-crystal molecules have a better planar alignment along the groove direction for the surfaces with smaller spatial periods of grooves, as shown in Figure 3.

The microgrooved polyimide PIA-5310 surfaces are also fabricated with a fixed spatial period but at different groove depths. It is found that the anchoring energy increases along with the groove depth, as shown in Table 1, consistent with the prediction of Berreman. ${ }^{20}$ In addition, Paek et al. ${ }^{6}$ also confirmed

(23) Bryan-Brown, G. P.; Sage, I. C. Liq. Cryst. 1996, 20, 825. this observation who manipulated mechanical rubbing on polyimide alignment layers with different pile impressions (the maximum depth of the rubbing cloth when pressed down by the roller). He found that the anchoring energy increases monotonically along with pile impression, indicating that the interaction between the rubbed polyimide surface and the liquid-crystal molecules is enhanced with larger rubbing strength. Our result is in contrast to that of Rastegar et al. ${ }^{24}$ These authors demonstrated that the anchoring energy of the atomic force microscope scanned polyimide surfaces is independent of the vertical scan force, that is, equivalent to the groove depth. Our result implies that the groove effect is indeed responsible for the uniform alignment of the nematic liquid crystal along the groove direction on the microgrooved polyimide surfaces fabricated by soft embossing process.

The anchoring energy increases along with the groove depth and decreases with an increase in the spatial period of grooves. It is thus plausible to observe good planar alignment along the groove direction at large groove depths and poor alignment along the groove direction at small groove depths under the condition of a fixed spatial period of grooves. Consequently, it is possible to extend the lateral correlation length of liquid-crystal molecules in the confinement by simply increasing the groove depth. The microgrooved polyimide PIA-5310 surfaces are further fabricated under the condition of a fixed spatial period $(6 \mu \mathrm{m})$ and a fixed groove width $(3 \mu \mathrm{m})$ but at three different groove depths. They are 60,330 , and $1630 \mathrm{~nm}$. It is found that the planar alignment of 5CBs along the groove direction becomes more uniform with larger groove depth, as shown in Figure 4. There are microdomains for small groove depths, say 60 and $330 \mathrm{~nm}$, and the director of the liquid crystals does not uniformly align along the direction of microgrooves, as shown in Figure $4 \mathrm{a}$ and $4 \mathrm{~b}$. When the groove depth is increased to $1630 \mathrm{~nm}$, the liquid crystal uniformly aligns along the groove direction. When the liquid-crystal molecules are confined in the microgrooves, even the groove width up to $3 \mu \mathrm{m}$, this confinement effect would induce the director of the liquid crystals to uniformly align along the groove direction. This observation is consistent with the results of Behdani et al. ${ }^{25}$ who pointed out that the lateral correlation length of $5 \mathrm{CBs}$ on atomic force microscopy nanolithographic ITO films is $2.2 \mu \mathrm{m}$. The groove depth of the microgrooved surfaces plays an important role in the uniformity of the planar alignment of the nematic liquid crystals along the groove direction. The lateral correlation length of $5 \mathrm{CB}$ s could be extended to $3 \mu \mathrm{m}$ by the compensation effect of the deep groove depth in this work.

On the other hand, the effect of the groove depth on the homeotropic alignment had been discussed previously. ${ }^{4}$ It was found that the liquid-crystal 5CB exhibits homeotropic alignment on surfaces of small groove depths under the condition of fixed spatial period and groove width. When the groove depth is further increased, there is a transition of the liquid-crystal alignment from homeotropic to planar. ${ }^{4}$

(24) Rastegar, A.; Škarabot, M.; Blij, B.; Rasing, Th. J. Appl. Phys. 2001, 89 960.

(25) Behdani, M.; Rastegar, A.; Keshmiri, S. H.; Missat, S. I.; Vlieg, E.; Rasing, Th. Appl. Phys. Lett. 2002, 80, 4635. 

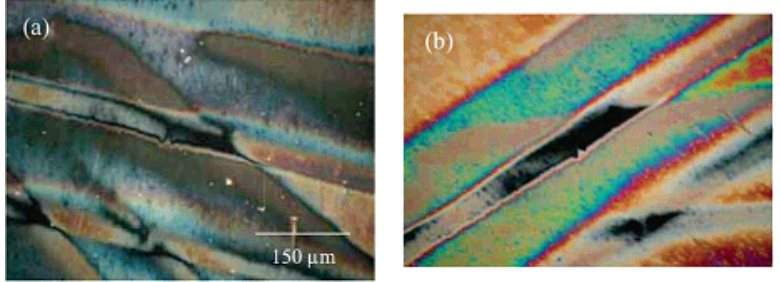

Figure 5. 5. Optical micrographs of liquid-crystal alignment on the mixed polyimide microgrooved surfaces with $R_{\mathrm{v}}=175$ taken between crossed polarizers. (a) The groove direction parallel to one polarizer axis; and (b) the groove direction at $45^{\circ}$ to each polarizer axis.

The polyimide PIA-5310 microgrooved surface can uniformly align $5 \mathrm{CBs}$ along the groove direction. Furthermore, it is important to have an appropriate pretilt angle of liquid crystals on an alignment layer to meet the requirement of electrooptical characteristics for the application in liquid-crystal display industry. It is found that the pretilt angle of $5 \mathrm{CBs}$ on the polyimide PIA5310 microgrooved surfaces is only $0.3^{\circ}$, a rather small value. The pretilt angle can be adjusted by tuning the surface polarity of the polyimide alignment layers.

In this work, the surface polarity of the polyimide surfaces is modified by simply mixing two kinds of polyimides: one is the planar alignment type (PIA-5310) and the other one is the homeotropic alignment type (JSR-2021). The alignment layers of PIA-5310 and of JSR-5310 are fabricated by the soft embossing method to generate the periodical microgrooves with $360-\mathrm{nm}$ groove width, 480-nm line width, and 360-nm groove depth. The pretilt angles $\left(\theta_{\mathrm{p}}\right)$ on the PIA-5310 and JSR-5310 alignment layers are $0.3^{\circ}$ and $90.0^{\circ}$, respectively. On the other hand, the advancing contact angles $\left(\theta_{\text {a }}\right)$ of water on flat polyimide surface of PIA-5310 and JSR-5310 are $67^{\circ}$ and $99^{\circ}$, respectively. Indeed, the PIA-5310 polyimide is more hydrophilic than that of the JSR-2021.

Then, two prepolymers PIA-5310 and JSR-5310 are mixed at different volume ratios to fabricate the alignment layers with periodical microgrooves by soft embossing method. The volume ratio $R_{\mathrm{v}}$ is defined by $R_{\mathrm{v}}=V_{\mathrm{PIA}-5310} / V_{\mathrm{JSR}-2021}$. The surface polarity of the alignment layer is successfully manipulated. That is, the advancing contact angle of water on the mixed polyimide surfaces decreases along with increasing $R_{\mathrm{v}}$. Table 2 lists the advancing contact angles $\left(\theta_{\mathrm{a}}\right)$ of water and the corresponding pretilt angles $\left(\theta_{\mathrm{p}}\right)$ of 5CBs on the surfaces fabricated from the mixtures of the prepolymers PIA-5310 and JSR-2021 at different $R_{\mathrm{v}}$ 's. The advancing contact angles of water on the mixed polyimide surfaces remain unchanged until $R_{\mathrm{v}}$ exceeds 100 . It is believed that during the thermal curing process, more hydrophobic JSR-2021 tends to have direct contact with the PDMS mold, which is very hydrophobic, and more hydrophilic PIA-5310 would orient toward inside the bulk phase of the alignment layer avoiding direct contact with the PDMS mold. Hence, when $R_{\mathrm{v}}$ is smaller than 100 , the surface is still fully covered by the hydrophobic polyimide JSR2021 , and the advancing contact angle of water on these surfaces does not change and remains $99^{\circ}$. However, when the amount of PIA-5310 is further increased, the amount of hydrophobic JSR-2021 is not enough to cover the whole surface, leading to a decrease in the advancing contact angle of water on the mixed polyimide surface.

Next, the soft embossing method is applied to fabricate the microgrooved surfaces with mixed prepolymers of PIA-5310 and JSR-2021 at different $R_{\mathrm{v}}$ 's to generate the alignment layers of different surface polarities. As shown in Table 2, the pretilt angle is indeed adjustable by the change of the surface polarity. (a)

(a')

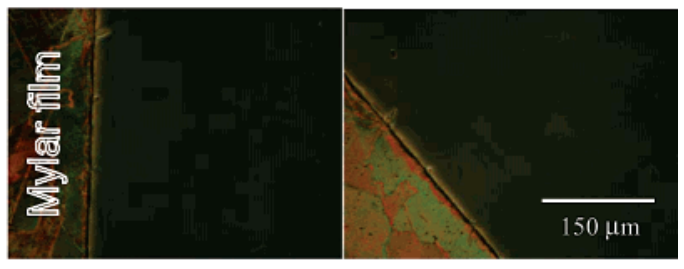

(b)

(b')

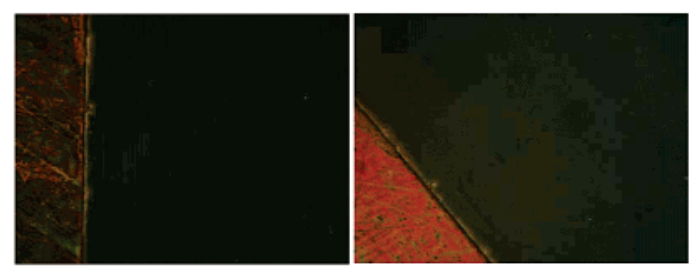

(c)

(c')

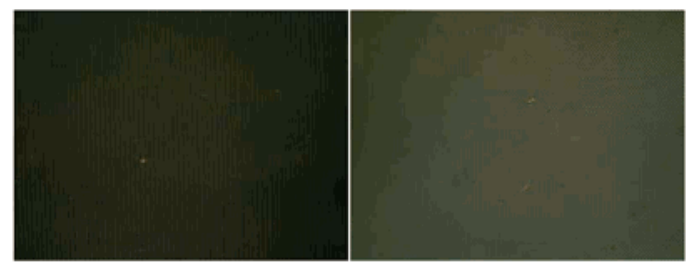

(d)

(d')

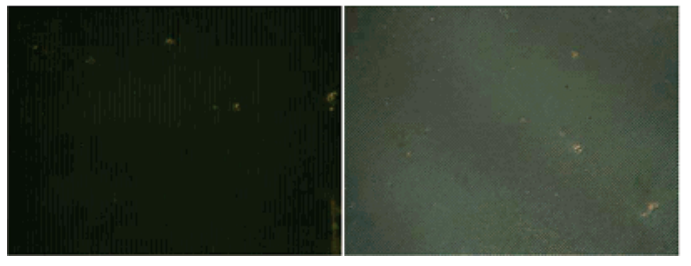

Figure 6. 6. Optical micrographs of liquid-crystal alignment taken between crossed polarizers for the patterned polyimide JSR-2021 surfaces with $6-\mu \mathrm{m}$ groove period and $3-\mu \mathrm{m}$ groove width at four different grating depths: $60 \mathrm{~nm}$ ( $\mathrm{a}$ and $\left.\mathrm{a}^{\prime}\right), 330 \mathrm{~nm}$ (b and $\left.\mathrm{b}^{\prime}\right), 900$ $\mathrm{nm}\left(\mathrm{c}\right.$ and $\left.\mathrm{c}^{\prime}\right)$, and $1630 \mathrm{~nm}\left(\mathrm{~d}\right.$ and $\left.\mathrm{d}^{\prime}\right)$. All the micrographs on the left-hand side, a, b, c, and d, are taken with the groove direction parallel to one of the polarizer axes, and all the micrographs on the right-hand side, $\mathrm{a}^{\prime}, \mathrm{b}^{\prime}, \mathrm{c}^{\prime}$, and $\mathrm{d}^{\prime}$, are taken with the groove direction at $45^{\circ}$ to each polarizer axis.

Furthermore, the pretilt angle increases along with the advancing contact angle of water on the surfaces. However, when $R_{\mathrm{v}}=175$ and 250, the alignment of liquid-crystal molecules is poor, as shown in Figure 5, and the pretilt angle is not measurable. The exact reason of poor alignment at $R_{\mathrm{v}}=175$ and 250 is unclear and is still under examination. It is very likely that the hydrophobic JSR-2021 aggregates to form microdomains instead of homogeneously distributing on the alignment layer surfaces, resulting in poor alignment of liquid-crystal molecules.

In addition, we would like to demonstrate that the topographical effect could also affect the pretilt angle of the supported liquid crystals on alignment layers of a weak polarity. It is mentioned above that the liquid-crystal $5 \mathrm{CB}$ aligns vertically on the polyimide JSR-2021 surface. Since the advancing contact angle of water on the polyimide JSR-2021 surface is $99^{\circ}$, the polarity of this surface is relatively weak. The microgrooved polyimide JSR2021 surfaces with $6-\mu \mathrm{m}$ spatial period and $3-\mu \mathrm{m}$ groove width 
are prepared on glass substrates at four different groove depths. There is no light passing through the cell of the groove depth at 60 or $330 \mathrm{~nm}$ when the micrographs are taken with the groove direction either parallel or at $45^{\circ}$ to one of the polarizer axes, as shown in Figure $6 \mathrm{a}$ and $6 \mathrm{~b}$. That implies the 5CBs exhibit homeotropic alignment on these surfaces. When the groove depth is further increased, there is a transition of the alignment of $5 \mathrm{CBs}$ from homeotropic to planar, as shown in Figure $6 \mathrm{c}$ and $6 \mathrm{~d}$. This observation indicates that when the surface topography is weak, the surface polarity dominates the alignment of 5CBs. On the other hand, when the surface topography gets more pronounced, it becomes the primary factor for the alignment of $5 \mathrm{CBs}$. Therefore, the liquid-crystal alignment is simply the outcome of the competition of the surface topography and the surface polarity.

\section{Conclusion}

The soft embossing method is proposed to fabricate the microgrooved polyimide surface for the liquid-crystal alignment layer. It is found that the liquid-crystal $5 \mathrm{CB}$ would have better planar alignment along the groove direction when the spatial period of groove is shorter and the groove depth is greater. It is also demonstrated that the pretilt angle of liquid crystals is the outcome of the competition between the surface topography and the surface polarity. The pretilt angle of liquid crystals increases along with the advancing contact angle of water, an index of the surface polarity, under the condition of a fixed surface topography.

LA061875F 